\title{
Lidil
}

Revue de linguistique et de didactique des langues

\section{Approche empirique de l'intercompréhension : répertoires, processus et résultats}

Raphael Berthele et Amelia Lambelet

\section{OpenEdition}

\section{Journals}

Édition électronique

URL : http://journals.openedition.org/lidil/2749

DOI : $10.4000 /$ lidil.2749

ISSN : $1960-6052$

\section{Éditeur}

UGA Éditions/Université Grenoble Alpes

\section{Édition imprimée}

Date de publication : 1 mai 2009

Pagination : 151-162

ISBN : 978-2-84310-139-7

ISSN : 1146-6480

\section{Référence électronique}

Raphael Berthele et Amelia Lambelet, « Approche empirique de l'intercompréhension : répertoires, processus et résultats », Lidil [En ligne], 39 | 2009, mis en ligne le 01 décembre 2010, consulté le 20 avril 2019. URL : http://journals.openedition.org/lidil/2749; DOI : 10.4000/lidil.2749 


\title{
Approche empirique de l'intercompréhension : répertoires, processus et résultats
}

\author{
Raphael BeRTHELE et Amelia LAMBELET*
}

\section{RÉSUMÉ}

Cet article présente une recherche sur les processus d'intercompréhension mis en œuvre pour la compréhension d'un texte dans une langue non ou peu apprise mais typologiquement proche d'une langue connue. Se basant sur la vision holistique du plurilinguisme, selon laquelle chaque langue participe à la compétence globale de communication, les auteurs cherchent à déchiffrer les mécanismes qui permettent d'inférer la signification d'items d'un texte dans une langue inconnue ainsi que sa compréhension globale et détaillée. Les principales pistes explorées sont la proximité linguistique des items et le profil linguistique des sujets.

\section{ABSTRACT}

In this contribution we discuss a study of interlingual inferencing processes as the ones typically applied in intercomprehension tasks. Based on the assumption that bi-and multilingual competence has to be regarded as a dynamic and holistic ensemble of interacting partial competences, the informant's capability of correctly inferring meanings of words and texts is quantitatively assessed. The role of linguistic distance between target words in unknown but typologically familiar (Romance) languages and the cognates in the first and second languages of the informants is assessed, as well as the contribution of individual types of bi-or multilingualism to the interlingual inferencing task.

* Université de Fribourg $(\mathrm{CH})$. 


\section{Plurilinguisme, enseignement des langues et intercompréhension}

Dans une Europe pluriculturelle et plurilingue, la question de la formation des enseignants à la diversité revêt une importance capitale. Au-delà des questions que pose l'interculturalité en termes d'identité, de représentations ou de patrimoines, notre article se focalise sur la gestion du plurilinguisme et de l'enseignement des langues à l'école, dans une visée de décompartimentalisation des compétences langagières et d'accentuation de l'importance des compétences réceptives. Lorsque l'on parle d'enseignement des langues dans une optique de plurilinguisme, il devient en effet nécessaire de souligner le fait que la compétence linguistique d'un individu bi-plurilingue n'est pas segmentée entre ces différentes langues mais que celles-ci s'imbriquent et se complètent. Il est donc important de s'intéresser aux compétences linguistiques des apprenants en tenant compte de leur diversité. Ceci peut prendre différentes formes passant de l'ouverture aux langues de la classe - implicitement par le travail sur les erreurs ou « fautes de langues » vues non pas comme un problème mais comme la preuve du travail de transfert de structures d'une langue à l'autre, ou explicitement à l'aide de programmes tels que l'éveil aux langues (Candelier, 2003) - à l'enseignement coordonné des langues qui vise à favoriser l'économie cognitive des étudiants en basant l'apprentissage sur des ressources déjà acquises ou en cours d'acquisition dans une autre branche (Gelmi et Saxalber, 1992 ; Wokusch et al., 2007 ; Wokusch, 2008).

D'autres pistes d'action en vue de parvenir à une compétence plurilingue sont issues des réflexions sur les compétences partielles - compétences productives à l'oral/à l'écrit ; compétences réceptives à l'oral/à l'écrit. Auparavant, l'enseignement des langues visait le développement de compétences dans la langue cible le plus proche possible d'une compétence idéalisée du locuteur natif monolingue. Or, si l'on réfléchit à la compétence linguistique dans une optique de plurilinguisme, on se rend compte aujourd'hui que cette visée n'est pas pertinente. En effet, si la compétence linguistique d'un locuteur plurilingue est le résultat des interactions entre ses compétences dans les différentes langues, celles-ci évoluent tout au long de son parcours biographique (Degache, 2006). Travailler sur les compétences partielles permet donc de construire une compétence plurilingue naturelle et adaptée à la biographie linguistique de chacun, en tablant « sur l'existence d'un niveau minimal permettant d'entretenir la motivation pour l'apprentissage des langues tout au long de la vie» (Matthey, 2005, p. 144). 
Notre étude sur la compréhension de langues typologiquement proches se situe dans ce paradigme de compétences partielles, plus particulièrement dans la perspective où les compétences réceptives sont essentielles, car elles permettent de développer l'intercompréhension, que nous définirons, en suivant Degache (2006), comme le fait de « comprendre la langue de l'autre et se faire comprendre » que ce soit à l'oral ou à l'écrit. Enseigner la « compréhension » ou « l'intercompréhension » implique d'appréhender les processus qui permettent l'accès au sens de la langue de l'autre, et les accroches sur lesquelles l'apprenant se base pour construire sa compétence de compréhension. L'étude présentée ici tente de se rapprocher de ces mécanismes par la mise en situation de lecture d'un texte dans une langue non apprise mais génétiquement proche de la L1 ou d'une autre langue apprise et analyse les facteurs facilitateurs ou perturbateurs pour la compréhension.

\section{Une étude empirique : questions de recherche et méthodologie}

Comme nous l'avons décrit ci-dessus, la pratique de l'intercompréhension peut être vue comme faisant partie d'une perspective holistique sur le répertoire plurilingue et son usage dynamique dans la communication et l'interaction. En particulier dans le monde des langues romanes, on trouve un nombre impressionnant de matériaux et d'outils didactiques visant à développer les compétences « intercompréhensives » (EuRom 4, Blanche-Benveniste et Valli, 1997 ; Galatea et Galanet, voir Dabène, 2003 ; EuroComRom/Germ, voir Meissner, Meissner, Stegmann et Klein, 2004). En parcourant ces ouvrages, il devient évident que l'outil principal pour « déplomber » le code d'une langue cible pas ou peu maitrisée est celui des transparences (ou correspondances) inter-langues, que ce soit au niveau structurel (modèles syntaxiques plus ou moins identiques), lexical, morphologique, ou phonologique/graphématique. Par conséquent, les méthodes visant à enseigner l'intercompréhension sont souvent truffées de tableaux et de listes comparatives. Bien entendu, cet accent sur les transferts positifs et (quasi) gratuits d'une langue à l'autre est l'approche la plus évidente du travail intercompréhensif. Néanmoins, le travail du linguiste comparatif ne correspond pas forcément aux besoins ni aux processus d'identification inter-langues et de compréhension des apprenants plurilingues qui ont pour tâche de déchiffrer des textes en langues peu ou pas apprises/acquises. Certaines correspondances 
- notamment au niveau des internationalismes - sont tellement évidentes qu'il est probablement inutile de les faire figurer dans des listes interminables (voir par exemple certaines listes dans Meissner et al., 2004). D'autres correspondances, bien que très systématiques, restent indécelables en raison de graphies ou prononciations difficiles. Et, en fin de compte, il est tout à fait imaginable qu'il existe des éléments dits de «profil » (Meissner et al., 2004, p. 255), qui, malgré leur statut d'éléments opaques, ne posent pas de problème dans le traitement d'un texte en langue inconnue. En d'autres termes, la perspective purement comparatiste sur les systèmes plus ou moins parallèles est une partie importante mais insuffisante dans la modélisation du processus d'intercompréhension.

Il nous semble donc important d'approfondir nos connaissances des processus d'identification inter-langues par des locuteurs plurilingues. Les trois tâches expérimentales discutées ci-dessous représentent une première approche visant à élucider ce processus. Trois questions de recherche ont guidé notre enquête :

1) Quel type d'éléments lexicaux peut être identifié/inféré spontanément dans une langue inconnue mais typologiquement proche d'une/de plusieurs langues parlées par un locuteur plurilingue ?

2) Dans quelle mesure des textes dans une telle langue sont-ils compréhensibles de manière spontanée?

3) Y a-t-il des profils plurilingues qui offrent des bases particulièrement favorables à la compréhension spontanée en langue inconnue?

Nous avons mené une enquête parallèle ciblant des langues romanes d'un côté et des langues germaniques de l'autre, mais pour des raisons d'espace nous nous concentrerons ici sur les langues romanes (voir Berthele, 2008, pour le versant germanique).

Un total de 140 participants, tous étudiants en psychologie (programme de BA (licence) francophone de l'Université de Fribourg/ Suisse) ont participé à cette expérience. Les étudiants parlent en moyenne 4,29 langues. La L1 de la plus grande majorité est le français, mais il y a aussi un groupe assez important d'italophones $(\mathrm{N}=32)$ ainsi que quelques locuteurs d'autres langues (allemand $\mathrm{N}=13$, autres $\mathrm{N}=8$ ). Les étudiants ont rempli un questionnaire qui vise à refléter leur répertoire verbal moyennant des échelles pour les compétences réceptives et productives en L1, L2, [...] Lx. En suite de quoi, les participants ont exécuté les trois tâches que nous présentons ci-dessous, avec une limite de temps imposée : 
Tâche A - Lecture de texte et traduction de mots avec contexte (15') Traduction d'une liste de 17 mots en Romontsch Sursilvan (une variété de romanche), faisant partie d'un article de journal en Sursilvan (cf. extrait 1) sur un attentat terroriste à Eilat (Israël). Pour chaque mot, les participants ont dû proposer une traduction et noter les liens interlangues ou les éléments contextuels leur ayant permis de traduire (cf. exemple 2).

(1) Morts pervia d'in attentat el liug da far bogn israelit Eilat AP $-29 / 01 / 2007^{1}$

Gerusalem - Gliendisdis ein treis persunas vegnidas per la veta pervia d'in attentat da suicidi el liug da far bogn israelit Eilat alla Mar Cotschna. Quei ha la polizia communicau. Tenor las medias israelitas ei igl attentat vegnius fatgs en ina pasternaria en in center da cumpra el quartier dil marcau Isidor. [...]

(2) Item : «liug »

Votre traduction du mot : «lieu»

Comment avez-vous deviné la signification du mot? En fonction du contexte ? À partir d'une autre langue/dialecte ? Si oui, précisez quelle autre langue ou dialecte vous y a aidée.

(2a) : « liug », « via luogo (Italien) - liug » (participant \#291);

(2b) : « regarder »; « dialecte suisse allemand "luege" » (\#301);

(2c) : « ligue »; «parce que le sujet de l'attentat doit être dans la première ligne $->$ liug = ligue $»(\# 280)$.

L'exemple 2a présente le cas d'une inférence correcte en utilisant des connaissances de l'italien. L'exemple $2 \mathrm{~b}$ présente une inférence erronée, à partir d'un faux ami suisse allemand. L'exemple $2 \mathrm{c}$ montre une inférence également erronée, mais celle-ci n'est pas basée sur une base de transfert lexicale mais sur des règles contextuelles.

Tâche B : Compréhension de texte (5')

Cette tâche vise à opérationnaliser la capacité de comprendre le texte (extrait 1) par le biais de sept questions de compréhension détaillées sous la forme de QCM avec quatre propositions de réponses.

Tâche C : Traduction sans contexte (10')

Cette tâche ressemble à la tâche $\mathrm{A}$, mais cette fois les 29 mots à traduire sont présentés hors contexte. Il s'agit uniquement de verbes très fréquents (mélange de roumain et de romontsch sursilvan), les participants

1. Nous remercions Renzo Caduff de son aide pour la traduction de cet article du néerlandais en Romontsch Sursilvan. 
étant informés de cette restriction. La liste comporte 29 items qui sont tous (théoriquement) plus ou moins transparents - c'est-à-dire qu'elle ne contient pas de mots «profil » (voir Meissner et al., 2004).

(5) Exemples tâche $\mathrm{C}$ - traduction de verbes hors contexte :

a) mirar -> « regarder », « via l'espagnol » (\#269; traduction correcte);

b) asculta $->$ « déculpabiliser», « via espagnol : culpa » (\#253; traduction correcte : « ausculter », « écouter»);

c) trer $->$ « essayer », « via l'anglais, try » (\#212; traduction correcte :

« tirer »).

Comme le montre l'exemple (5b), l'évaluation des réponses doit être relativement tolérante face aux glissements sémantiques, sinon cette tâche hors contexte serait insoluble.

\section{Résultats}

Afin de répondre à la première question concernant le type d'éléments qui peut être inféré sans grandes difficultés, nous avons calculé la distance graphématique entre le mot en langue cible et son mot apparenté en français et/ou italien. Puisqu'il y a beaucoup de participants avec de bonnes voire très bonnes compétences en italien dans notre échantillon, nous avons décidé de prendre en compte l'italien comme langue de base ou langue pont. La distance a été calculée en utilisant une mesure très simple, celle de Levenshtein (aussi dénommée EDIT-distance ; Levenshtein, 1966). Vu la nature purement écrite de nos tâches, nous n'avons pas osé émettre des hypothèses concernant la phonologie (imaginée) des mots en langue cible inconnue et sa proximité par rapport aux mots apparentés dans le répertoire plurilingue des participants. C'est pourquoi nous avons calculé la distance de Levenshtein pure, c'est-à-dire la distance qui calcule simplement le nombre de caractères à changer afin d'arriver du mot cible au mot servant (potentiellement) de base de transfert. Puisque nous travaillons dans une logique de comparaison de graphèmes et non pas avec des formes phonologiques, il est difficile de peser des proximités phonologiques comme le font les méthodes utilisées en dialectométrie quantitative (voir Heeringa et al., 2006, pour une comparaison de méthodes comparatives quantitatives). En d'autres termes, la proximité évidente (bien qu'elle reste sur un niveau d'une phonologie « hypothétique » ou imaginée) de deux caractères, par exemple $<0>$ et $<\mathrm{a}>$ par opposition à la distance entre $<0>$ et $<\mathrm{r}>$, n'est donc pas prise en compte. Ceci présente un désavantage 
significatif, mais pour le moment incontournable, lié à la méthodologie choisie pour cette expérience (cf. la discussion des résultats ci-dessous). L'exemple (6) illustre cette manière - forcément grossière - de calculer la proximité de deux paires de mots :

(6a) cugeta (roum.)

cogiter (fr), cogitare (it) ; distance « EDIT » : 4 (pour it et fr), distance normalisée (par rapport à la longeur du mot le plus long) : 4/7 =0,57

(fr), $4 / 8=\mathbf{0 , 5}$ (it)

(6b) buc (romontsch)

pas, non ou ne (fr), non (it) ; distance «EDIT » : 3 (pour it et fr), distance normalisée : $\mathbf{1}$ (pour it et fr)

Cette manière de calculer les distances est certes très approximative si l'on tient compte de son incapacité totale à prendre en considération les distances phonologiques très variables des segments édités, mais a néanmoins l'avantage d'être neutre par rapport à des décisions souvent arbitraires concernant le statut de mot apparenté (ou cognat) ou mot non-apparenté (ou mot profil). En plus, la méthode permet de situer les paires de mots sur une échelle entre totalement transparent (distance 0) et opacité totale (distance 1).

Le tableau 1 présente tous les mots avec leur distance Levenshtein/ EDIT par rapport à l'italien ou au français (en choisissant toujours la valeur la plus basse des deux). Les items sont présentés par ordre décroissant de la proportion d'inférences correctes de la part de nos participants.

\begin{tabular}{|l|c|c|l|c|c|}
\hline Item & EDIT & $\begin{array}{c}\text { inférences } \\
\text { correctes }\end{array}$ & Item & EDIT & $\begin{array}{c}\text { inférences } \\
\text { correctes }\end{array}$ \\
\hline menader & 0,38 & 0,56 & marcau & 0,33 & 0,03 \\
\hline liug & 0,40 & 0,53 & endisch & 0,43 & 0,02 \\
\hline tenor & 0,60 & 0,38 & aschibein & 0,45 & 0,01 \\
\hline malfatgs & 0,50 & 0,37 & onn & 0,50 & 0,01 \\
\hline pasternaria & 0,36 & 0,32 & cartents & 0,44 & 0,00 \\
\hline dapi & 0,50 & 0,28 & davos & 0,83 & 0,00 \\
\hline inimitg & 0,25 & 0,11 & quintaus & 0,63 & 0,00 \\
\hline piarder & 0,38 & 0,07 & siglientau & 0,66 & 0,00 \\
\cline { 1 - 2 } unfrendas & 0,89 & 0,04 & \multicolumn{5}{l}{} &
\end{tabular}

Tableau 1. - Distance Levenshtein / EDIT et proportion d'inférences correctes. 
Le tableau montre qu'il n'y a qu'un lien relativement faible entre la distance graphique inter-langues et la capacité d'inférer le sens en contexte. Il y a des mots très peu transparents (comme par exemple «tenor »- «selon »- «secondo ») qui sont malgré tout relativement bien inférables en contexte. À l'inverse, il y a aussi des mots relativement transparents («cartents»- «croyants»- «credenti $»)$ qui ne sont jamais traduits correctement par nos participants. Ceci souligne d'un côté le rôle important du contexte et son potentiel d'enrichissement et de compensation des «trous lexicaux » en situation de lecture en L2 et en intercompréhension, et de l'autre l'insuffisance du seul critère de proximité linguistique pour la compréhension de texte en langue apparentée. Une analyse statistique (corrélation pearson $r=-0,298$, p $>0,05)$ montre bien la corrélation négative attendue entre inférabilité (la capacité de nos participants à deviner les sens des mots en langue inconnue) et distance graphique, mais ce n'est qu'une tendance statistiquement non significative.

Le même traitement a été administré aux données de la tâche $\mathrm{C}$, les inférences inter-langues hors contexte. Nous nous attendions cette fois-ci à ce qu'il y ait un lien bien plus direct entre les correspondances de forme et les inférences de sens correctes, vu l'absence totale de contexte. Cette hypothèse est vérifiée $(r=-0,320, P<0,05)$. Le fait que le coefficient de corrélation reste malgré tout assez bas pourrait s'expliquer comme suit : d'une part par la manière de calculer la distance inter-langues relativement imprécise (voir ci-dessus), et d'autre part en raison de l'élément important mais pour le moment inexploré de la phonologie « imaginaire » que les participants attribuent aux mots écrits en langue inconnue et des associations correctes ou erronées qui se font au niveau de cette phonologie hypothétique. Des recherches plus approfondies visant à optimiser ces deux lacunes de la méthodologie appliquée dans notre étude semblent donc indiquées et prometteuses.

La compréhension du texte lui-même varie considérablement à l'intérieur de notre échantillon. Certaines personnes répondent correctement à toutes les questions, tandis que d'autres ne répondent correctement à aucune question. La moyenne est située à peu près à $40 \%$ de réponses correctes aux sept questions de compréhension du texte.

La dernière question qui sera traitée ici est de savoir s'il y a des profils particuliers de plurilingues qui sont particulièrement aptes à trouver ces correspondances inter-langues. On pourrait supposer par exemple que le simple fait de parler beaucoup de langues favorise aussi le «bricolage » inter-langues que représente l'intercompréhension. D'un côté 
ceci pourrait être dû au simple fait que le répertoire plurilingue est plus grand et présente plus de bases de transfert. De l'autre côté, on pourrait aussi émettre l'hypothèse que les plurilingues ont l'habitude d'être confrontés à des problèmes de compréhension en L2 ou Lx et qu'ils/ elles ont développé des stratégies efficaces inter- et translinguistiques qui favorisent encore une fois la tâche de l'intercompréhension.

En groupant nos informateurs selon différents types de profils plurilingues, nous avons essayé d'identifier les groupes d'individus les plus efficaces dans la solution des trois tâches présentées dans notre article. Le premier résultat est que nous pouvons en effet constater que les individus qui connaissent davantage de langues - en faisant abstraction, dans un premier temps, du niveau de compétence respectif - sont plus efficaces (nombre de langues et inférences correctes : spearman's rho $=0,159, \mathrm{p}<0,05$ pour la tâche A ; spearman's rho $=0,351, \mathrm{p}<0,01$ pour la tâche C). L'effet du plurilinguisme, ainsi opérationnalisé en tenant compte simplement du nombre de langues dans le répertoire déclaré, est bel et bien mesurable, mais il reste très faible. À partir des données du questionnaire sur le profil linguistique individuel, nous avons essayé de faire une analyse un peu plus différenciée visant à établir les liens mesurables entre répertoires plurilingues individuels et capacité « intercompréhensive » de nos participants. Nous avons donc construit d'autres groupes de participants, cette fois-ci en tenant compte des niveaux de compétences en deux ou plusieurs langues (cf. figure 1). Nous avons notamment comparé des groupes de personnes qui ont de très bonnes compétences dans au moins deux langues romanes (compétences supérieures au niveau 4 sur une échelle de 0 à 6) et celles qui ont de très bonnes compétences (en appliquant le même seuil) dans une langue romane et au moins une autre langue non-romane. Pour les tâches $\mathrm{A}$ et $\mathrm{C}$, cette analyse montre qu'il est clairement avantageux pour l'intercompréhension ciblant une langue romane inconnue d'avoir une bonne maîtrise de deux langues romanes. De l'autre côté, le simple fait d'être très compétent en deux langues (par exemple en français et en allemand ou en anglais) ne présente pas d'avantage mesurable pour la tâche de l'intercompréhension.

Ces différences sont statistiquement avérées dans une analyse qui utilise le modèle linéaire général en comparant les deux groupes des bilingues en langues romanes aux bilingues « tout court », donc sans tenir compte de la proximité linguistique des deux langues maitrisées à un niveau élevé (tâche $\mathrm{A}: \mathrm{F}=6,882, \mathrm{p}=0,010$ pour les bilingues « romans », $\mathrm{F}=2,033, \mathrm{p}<0,05$ pour les bilingues ; tâche $\mathrm{C}: \mathrm{F}=19,565$, 


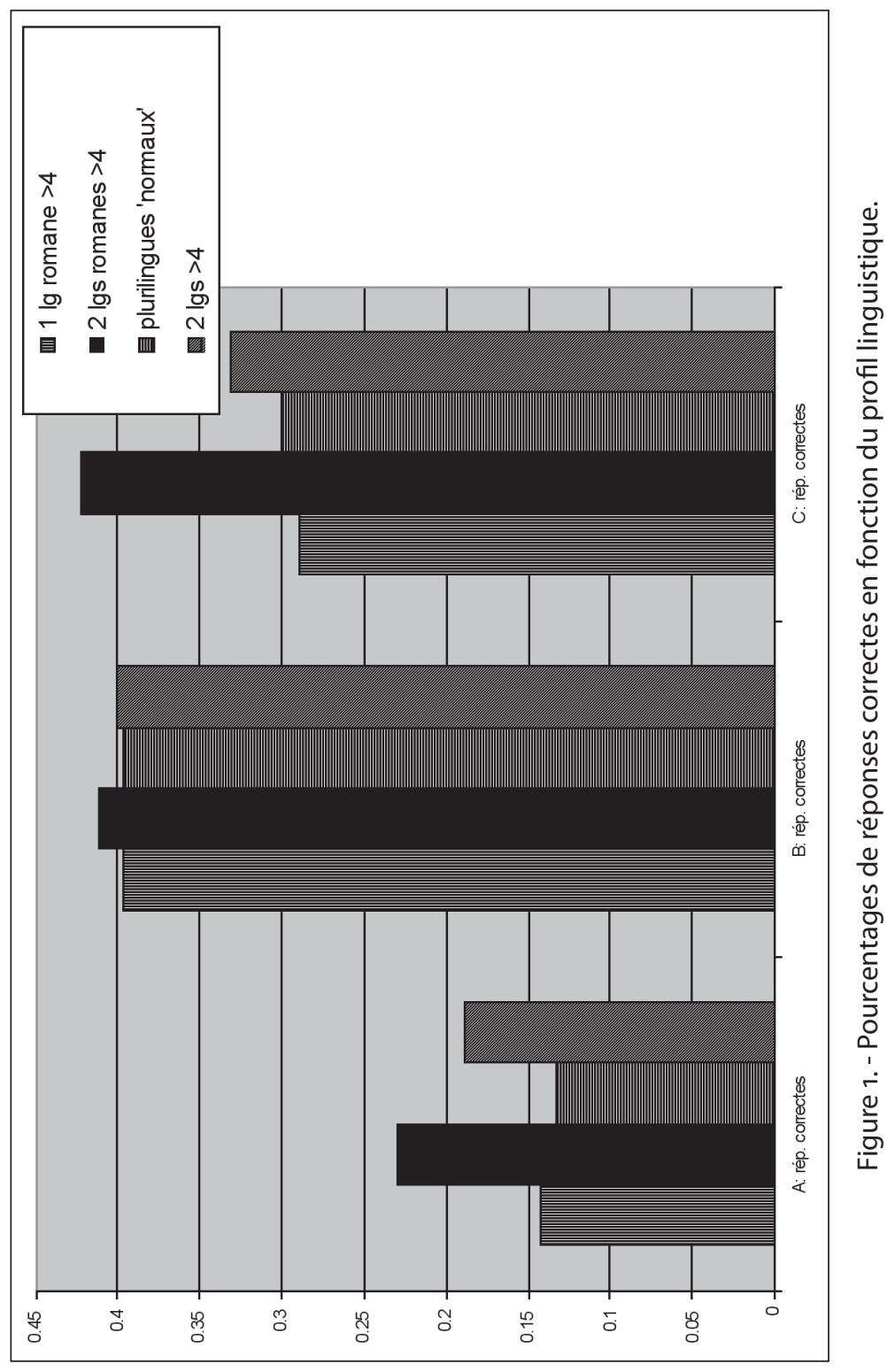


$\mathrm{p}<0,01$ pour les bilingues « romans », $\mathrm{F}=1,351, \mathrm{p}>0,05$ pour les bilingues). Le même résultat est obtenu dans l'étude strictement parallèle sur des langues cibles germaniques (Berthele, 2008) et il semblerait donc judicieux d'en conclure qu'une très bonne maitrise de deux langues apparentées donne naissance à une sensibilité accrue concernant les correspondances possibles et potentielles entre deux ou plusieurs systèmes apparentés. Berthele (2008) nomme cette capacité particulière pour trouver des correspondances inter-langues tolérance perceptive, une tolérance qui serait donc plus prononcée chez des plurilingues possédant deux systèmes proches bien développés.

\section{Conclusion}

Notre recherche ouvre une perspective micro sur les processus de l'intercompréhension et nous a permis de mettre en évidence les effets contrastés de la proximité linguistique, ainsi que le rôle du contexte pour la compréhension. Elle a aussi mis au jour certaines particularités en termes de profils linguistiques favorisant la compréhension dans une langue génétiquement proche. Ce qui est à première vue surprenant dans cette analyse est que la compréhension de texte en langue inconnue semble largement indépendante du répertoire plurilingue individuel. Ce résultat pourrait confirmer ce que nous savons de la compréhension des textes écrits, à savoir que celle-ci est influencée par le niveau d'acculturation à l'écrit - ce qu'on appelle parfois les compétences littératiées. Ces compétences font partie de ce que Cummins (1979) appelle la cognitive academic language proficiency. Elles comportent à la fois la capacité à utiliser des stratégies adaptées à la tâche de lecture (Alderson et Urquhart, 1992 ; Alderson, 2000 ; Anderson, 1991), mais aussi le degré de connaissance du contexte politico-géographique spécifique auquel l'article de journal fait référence.

\section{RÉFÉRENCES BIBLIOGRAPHIQUES}

Alderson J. C. (2000) : Assessing reading, Cambridge, CUP.

Alderson J. C., URquhart A. H. (dir.) (1992) : Reading in a foreign language, London, New York, Longman.

Anderson N. J. (1991) : «Individual differences in strategy use in second language reading and testing », Modern Language Journal, 75, p. $460-472$. 
Berthele R. (2008) : «Dialekt-Standard Situationen als embryonale Mehrsprachigkeit. Erkenntnisse zum interlingualen Potenzial des Provinzlerdaseins », dans K. J. Mattheier et A. Lenz (dir.), Dialektsoziologie / Dialect Sociology / Sociologie du Dialecte, Sociolinguistica, 22, p. 87-107.

Blanche-Benveniste C. et Valli A. (1997) : Eurom 4 - Méthode d'enseignement de quatre langues romanes, Firenze, Nuova Italia.

CANDELIER M. (2003) : Evlang - l'éveil aux langues à l'école primaire - Bilan d'une innovation européenne, Bruxelles, De Boeck Éditions.

Cummins J. (1979) : « Cognitive/Academic Language Proficiency, Linguistic Interdependence, the Optimum Age Question and some Other Matters », Working Papers on Bilingualism, 19, p. 197-205.

DAbÈne L. (2003) : « De Galatea à Galanet. Un itinéraire de recherche, Intercompréhension entre langues romanes », Lidil, ${ }^{\circ} 28$, p. 23-29.

Degache C. (2006) : Didactique du plurilinguisme, travaux sur l'intercompréhension et l'utilisation des technologies pour l'apprentissage des langues. Disponible sur <http://www.galanet.eu/publication/fichiers/ HDR2006_DegacheC.pdf> (consulté le 20/11/2008).

Gelmi R. et A. SAXalber (dir.) (1992) : Didattica : Linguistica integrata : Beiträge zu Erziehung und Unterricht in Südtirol, Bozen, Pädagogisches Institut.

Heeringa W., Kleiweg P., Gooskens C. et Nerbonne J. (2006) : «Evaluation of String Distance Algorithms for Dialectology », dans Proceedings of the Workshop on Linguistic Distances, Sidney, Australian Association for Computational Linguistics, p. 51-62.

Levenshtein V. I. (1966) : «Binary codes capable of correcting deletions, insertions, and reversals », Soviet Physics Doklady, 10(8), p. 707-710.

Matthey M. (2005) : «Plurilinguisme, compétences partielles et éveil aux langues. De la sociolinguistique à la didactique des langues », dans M. Pouliot, E. Boulea et J.-P. Bronckart (dir.) : Repenser l'enseignement des langues : comment identifier et exploiter les compétences, Villeneuve d'Ascq, Presses universitaires du Septentrion, p. 135-159.

Meissner F.-J., Meissner C., Klein H. G. et Stegmann T. D. (2004) : EuroComRom - Les sept tamis : lire les langues romanes dès le départ. Avec une introduction à la didactique de l'eurocompréhension, Aachen, Shaker.

Wокusch S. (2008) : « Didactique intégrée des langues : la contribution de l'école au plurilinguisme des élèves », Babylonia, 1, p. 12-14.

Wокusch S. et al. (2007) : La didactique intégrée des langues à l'école. Tentative de conceptualisation, Version 1, Travaux du Groupe de référence pour l'enseignement des langues en suisse romande (GREL), HEP de Lausanne, Université de Lausanne. 